\title{
NUMERICAL ANALYSIS OF THE DEEP DRAWING PROCESS INCLUDING THE HISTORY OF STRESS AND STRAIN
}

\author{
PAWEL KALDUnSKi \\ Koszalin University of Technology, Koszalin, Poland \\ e-mail: pawel.kaldunski@tu.koszalin.pl
}

\begin{abstract}
This paper discusses the results of a numerical study of circular cup drawing of steel sheets using finite element method. The drawing process is considered as a geometrical and physical nonlinear problem with unknown boundary conditions in the contact area of the system, such as the tool and the workpiece. The updated Lagrangian description is used to characterize these nonlinear phenomena on a typical incremental step time. Numerical results are obtained using an explicit method in Ansys/Ls-Dyna program. The constitutive Cowper-Symonds material model with linear hardening strain to predict material plasticity is used. The results of implementation of stresses and strains from a blanking operation flat disc of a sheet of metal for deep-drawing process are presented. After the blanking process simulation, an implicit springback analysis is performed. Then a numerical analysis of cup forming from this flat disc plate was carried out. The analysis results are compared with one another through reading of the sheet thickness in several characteristic points and the overall height of the product.
\end{abstract}

Keywords: deep drawing, modeling, numerical analysis, FEM, stress history, strain history

\section{Introduction}

At present, in metal forming industry, preparation of three-dimensional models for the drawing is very important to explain and to understand specific forming processes which occur in the drawpiece. There are many different methods of building finite element models of drawing examples using 2D numerical and analytical models (Yao and Cao, 2001). Currently, in the modelling and simulation of drawing processes, the influence on state of stress and strain prior treatments is mainly omitted (Nagasekhar et al., 2006; Trzepiecinski and Gelgele, 2011). Apart from the unknown state after sheet rolling (Kim and Olver, 1998), during the blanking process, additional stress is placed on the disc periphery. The blanking process is understood as cutting out a flat disc from a flat metal sheet (Bohdal et al., 2014). As the tool, a die and a punch with cutting edges were used, and to prevent possible warping of the disc after cutting out, a blankholder was used (Urriolagoitia-Sosa et al., 2011). Then, the drawpiece without a flange was formed from the flat disc.

This paper presents the results of a comprehensive modelling of the drawing process including the history of stress and strain. The blanking process modelling has been carried out, which takes into account a springback phenomenon that occurs after the load is removed. Such an analysis allows a more accurate determination of the finished product dimensions, the drawing force in the whole process, and it permits one to determine the state of stress at any place of the drawpiece. From the mechanical perspective, this process is treated as a doubly nonlinear initial-boundary value problem with movable nonlinear sources and boundaries and only partial knowledge of the boundary conditions. The following nonlinearities occur in the process: geometric, physical and boundary conditions in the area of tool-sheet contact. The geometric nonlinearity, which is thought to be a nonlinear dependence between the strain and displacement, results from a change 
in the object geometry. The physical (i.e., material) nonlinearity is caused by nonlinearity of the mechanical properties of the material. A mathematical description of these nonlinear phenomena requires the use of rules regarding formulation of boundary and initial problems.

\section{Finite element modelling}

The description of nonlinearity of the material is conducted using an incremental model that takes into account the influence of the history of strains. The object is treated as a body in which elastic strains may occur (in the scope of reversible strains) together with plastic strains (in the scope of irreversible strains) with nonlinear hardening (Gambin and Kowalczyk, 2003). For the purpose of constructing a material model, the following are used: Huber-Mises-Hencky's nonlinear plasticity condition, the associated flow law and isotropic hardening (Jemiolo and Gajewski, 2014). The state of the material after the aforementioned processing is taken into account by introducing the following initial states: displacement, stresses and strains. The states of strains are described with nonlinear dependences and no linearization (Simo and Hughes, 1998). In this description, adequate measures are used for an increment of strains and for an increment of stresses (i.e., an increment of the Green-Lagrange strain tensor and an increment of the second Piola-Kirchhoff symmetric stress tensor). The incremental contact model covers contact forces, contact rigidity, contact boundary conditions and friction coefficients in this area. The mathematical model is supplemented with incremental equations of motion of the object and uniqueness conditions. An incremental function of the total energy of the system is introduced. From the stationary condition of this function, it is possible to derive a variational nonlinear equation to describe motion and deformation of the object for a typical incremental step. This equation is untangled with spatial discretization using the finite element method, which results in discrete systems of equations for motion and deformation of the object in the drawing process (Zienkiewicz and Taylor, 2006).

\subsection{Basic relationships}

Components of the Green-Lagrange strain tensor increment for a typical time step $\Delta t$ and for a non-linear isotropic material with mixed hardening are calculated from the formula (Bohdal and Kukielka, 2014; Bohdal, 2015)

$$
\Delta \varepsilon_{i j}=\frac{1}{1-\widetilde{S}^{* *}}\left(D_{i j k l}^{(E)} \Delta \sigma_{k l}-\frac{\frac{2}{3} \sigma_{Y}(\cdot) \dot{E}_{T} \Delta \dot{\varepsilon}_{e} \widetilde{S}_{i j}}{\widetilde{S}_{i j} C_{i j k l}^{(E)} \widetilde{S}_{k l}+\frac{2}{3} \sigma_{Y}^{2}(\cdot)\left(\widetilde{C}(\cdot)+\frac{2}{3} E_{T}\right)}\right)
$$

Components of the Piola-Kirchhoff stress tensor increment for a typical time step $\Delta t$ and for a non-linear material with mixed hardening are calculated using the formula

$$
\Delta \sigma_{i j}=C_{i j k l}^{(E)}\left(\Delta \varepsilon_{k l}-\psi \frac{\widetilde{S}_{k l}\left(\widetilde{S}_{i j} C_{i j k l}^{(E)} \Delta \varepsilon_{k l}-\frac{2}{3} \sigma_{Y}(\cdot) \dot{E}_{T}^{(V P)} \Delta \dot{\varepsilon}_{z}^{(V P)}\right)}{\widetilde{S}_{i j} C_{i j k l}^{(E)} \widetilde{S}_{k l}+\frac{2}{3} \sigma_{Y}^{2}(\cdot)\left(\widetilde{C}(\cdot)+\frac{2}{3} E_{T}\right)}\right)
$$

where $\psi$ is the load factor and is $\psi=1$ for loading and $\psi=0$ for unloading processes, $\widetilde{S}^{* *}=\widetilde{S}_{i j}^{*} C_{i j m n}^{(E)} \widetilde{S}_{m n}$ is a positive scalar variable, $\widetilde{S}_{i j}=S_{i j}-\alpha_{i j}(i, j=1,2,3)$ are the stress deviator components, $\alpha_{i j}$ are the translation tensor components, $\widetilde{\mathbf{D}}_{\sigma}$ and $D_{i j k l}^{(E)}$ are components of the tensor $\mathbf{D}^{(E)}=\mathbf{C}^{(E)^{-1}}$ in time $t, C_{i j k l}^{(E)}$ are the elastic constitutive tensor components $\mathbf{C}^{(E)}, \widetilde{C}(\cdot)=\widetilde{C}\left(\varepsilon_{e}^{(V P)}, \dot{\varepsilon}_{e}^{(V P)}\right)$ is the temporary translation hardening parameter in time $t$. $\sigma_{Y}(\cdot)=\sigma_{Y}\left(\varepsilon_{e}^{(V P)}, \dot{\varepsilon}_{e}^{(V P)}\right)$ is the accumulated material yield stress which depends on the history 
of viscoplastic strain and strain rate, $\varepsilon_{e}^{(V P)}$ and $\dot{\varepsilon}_{e}^{(V P)}$ are the cumulative effective viscoplastic strain and strain rates, respectively, $E_{T}$ is the strain hardening modulus at time $t, \dot{E}_{T}$ is the strain hardening modulus rate at time $t$ (Bohdal, 2015).

In the analysis of the deep drawing process, the bilinear model is used. This material model has a linear hardening relationship between stress and strain described with the empirical model: $\sigma_{Y}=\sigma_{Y 0}+E_{t a n} \varepsilon_{e q}^{(P)}$, where $\sigma_{Y 0}$ is the initial yield stress, $E_{t a n}=\left(E_{T} E\right) /\left(E-E_{T}\right)$ is the material parameter dependent on the modulus of plastic hardening $E_{T}=d \sigma_{Y} / d \varepsilon_{\text {eq }}^{(P)}$ and Young's elastic modulus $E, \varepsilon_{e q}^{(P)}$ is the equivalent plastic strain. The Cowper-Symonds elastic/viscoplastic material model is used for computer simulation of the cutting process. According to the strain criterion, material separation occurs when the strain value of the leading node is greater than or equal to a limiting value. The limiting strain used is $\varepsilon_{f}=1$. When an element of the matrix material reaches the limiting strain value, the corresponding element will be deleted. The Cowper-Symonds model allows for linear isotropic $(\beta=1)$ assumed in simulations, kinematic ( $\beta=0)$ or mixed $(0<\beta<1)$ plastic strain hardening, and the effect of the plastic strain velocity is given by the following power relation

$$
\sigma_{p}=\left(1+\frac{\dot{\varphi}_{i}^{(p)}}{C}\right)^{m}\left(R_{e}+\beta E_{p} \varphi_{i}^{(p)}\right)
$$

where $\beta$ is the plastic strain hardening parameter, $R_{e}[\mathrm{MPa}]$ is the initial static yield point, $\dot{\varphi}_{i}^{(p)}\left[\mathrm{s}^{-1}\right]$ is the plastic strain rate, $C\left[\mathrm{~s}^{-1}\right]$ is the material parameter defining the effects of the plastic strain rate, $m=1 / P$ is the material constant defining the sensitivity of the material to the plastic strain rate, $\varphi_{i}^{(p)}[-]$ is the plastic strain intensity, and $E_{p}=\left(E_{T} E\right) /\left(E-E_{T}\right)$ is a material parameter dependent both on the plastic strain hardening modulus, $E_{T}=\partial \sigma_{p} / \partial \varphi_{i}^{(p)}$, and Young's modulus E.

\section{Numerical model and results}

The blanking and drawing tools are treated as non-deformable bodies, i.e., $E \rightarrow \infty$. The following material parameters are assumed for DC01 deep-drawing steel (Kaldunski, 2009): Young's modulus $E=210 \mathrm{GPa}$, Poisson's ratio $\nu=0.29$, density $\rho=8000 \mathrm{~kg} / \mathrm{m}^{3}$, yield stress $R_{e}=200 \mathrm{MPa}$, tangent modulus $E_{\text {tan }}=1050 \mathrm{MPa}$.

In this study, the objects are meshed with an 8-node SOLID 164 element type with reduced integration and hourglass control. The sheet has been divided into cubic elements to eliminate the influence of non-uniform mesh on the final results. The contact between ideally rigid tools and the deformable sheet metal is described using Coulomb's friction model according to the formula

$$
\mu_{c}=F_{D}+\left(F_{S}-F_{D}\right) \mathrm{e}^{-D_{C}\left|v_{r e l}\right|}
$$

where $F_{D}$ is the dynamic friction coefficient, $F_{S}$ - static friction coefficient, $D_{C}$ - exponential decay coefficient, e - Euler's number, $v_{r e l}$ - relative velocity of the surfaces in contact.

The following coefficients of friction in the contact zone between the stamp and sheet have been adopted: static friction coefficient $\mu_{s}=0.2$, dynamic friction coefficient $\mu_{d}=0.1$.

The following coefficients of friction in the contact zone between the die block and sheet have been adopted: static friction coefficient $\mu_{s}=0.1$, dynamic friction coefficient $\mu_{d}=0.01$.

The $D_{C}$ exponential decay coefficient is 10 . The dependence of the coefficients of friction between the sheet and the stamp on the relative velocity of the surfaces in contact is presented with the solid line on the graph (Fig. 1), while the dotted line shows the dependence of the friction coefficients between the sheet and the die block. 


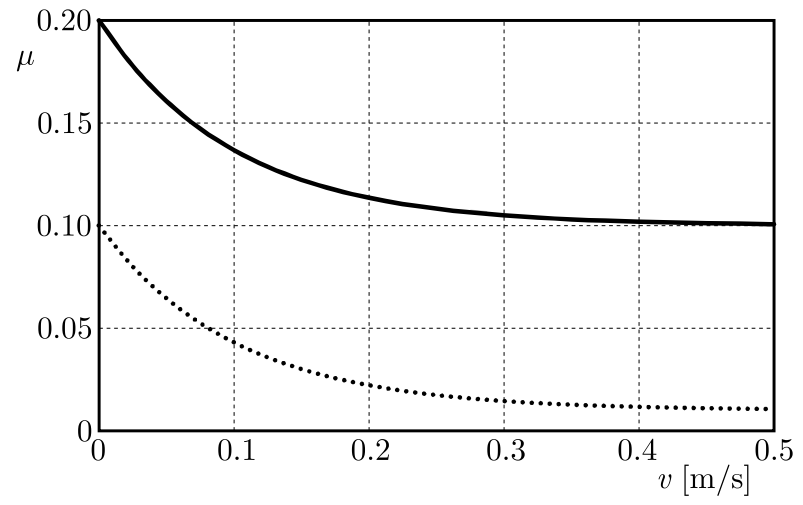

Fig. 1. Friction coefficient as a function of relative velocity of the surfaces in contact

An additional blankholder is applied from the bottom, which eliminates the bulge out effect of the blanked element. The type of the material used for this calculation is sheet metal stamping DC 01 with thickness $g=2 \mathrm{~mm}$. Diameter of the blanked disc $D=70 \mathrm{~mm}$. Figure 2 shows the initial mesh of the blanking process. The cutting areas of the die and punch are divided on a denser mesh to increase the calculation accuracy.

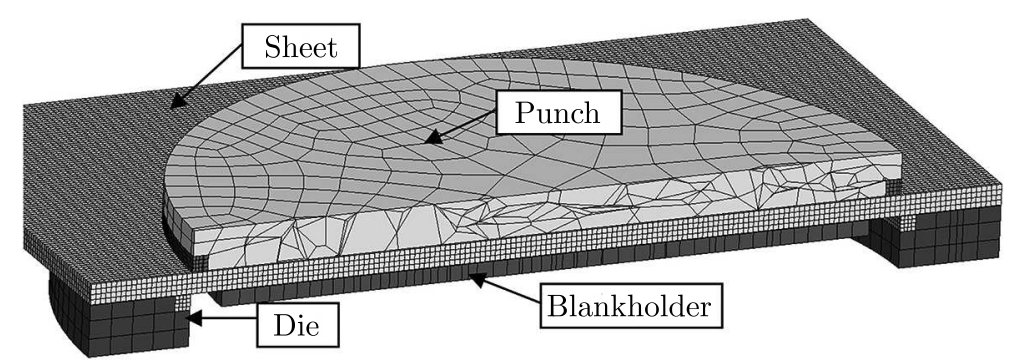

Fig. 2. Initial mesh of the blanking process

Figure 3 shows the equivalent stress distribution in the blanked disc. It may be observed that the maximum stress values are located on the disc periphery in the cutting area.

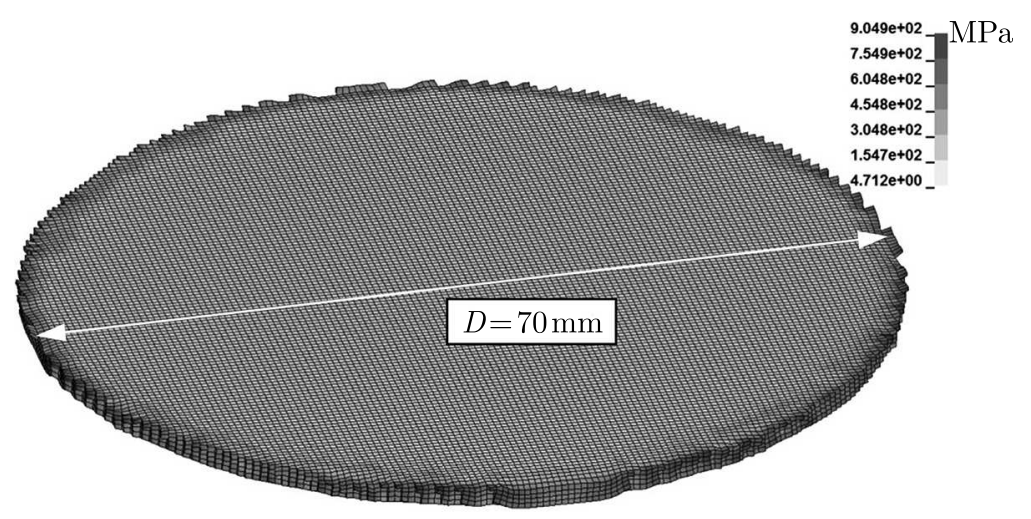

Fig. 3. Equivalent stress distribution in the blanked disc

Figure 4 shows the equivalent stress distribution in the disc after unloading. This analysis is necessary in a multistage process. Otherwise, in further simulations, it does not receive any correct results. It can be observed that the values of the equivalent stress at the periphery of the disc after removing the elastic strain is almost 3 times reduced. 


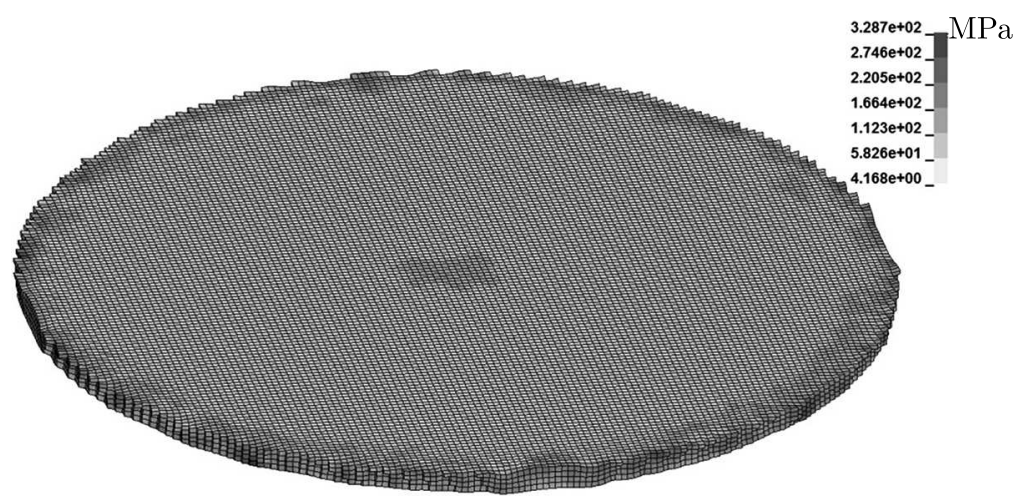

Fig. 4. Equivalent stress distribution in the blanked disc after springback

\subsection{Deep drawing without the history of stress and strain}

Figure 5 shows a cross-sectional view of a discrete model of the drawing process (Kaldunski and Kukielka, 2014). The die which is used has rounding radius $r_{d}=16 \mathrm{~mm}$ and internal diameter $d=40 \mathrm{~mm}$. The punch diameter has rounding radius $r_{p}=4 \mathrm{~mm}$ and its diameter is equal to $d_{p}=35 \mathrm{~mm}$. The clearance die is therefore $2.5 \mathrm{~mm}$. An acceptable reduction of the diameter in the drawing process determined by the $m_{1}$ coefficient for the ratio $D / g=35$ equals $m_{1}=0.5$ (Table 1 ). This means that the value $m_{1}=d / D=0.57$ is acceptable.

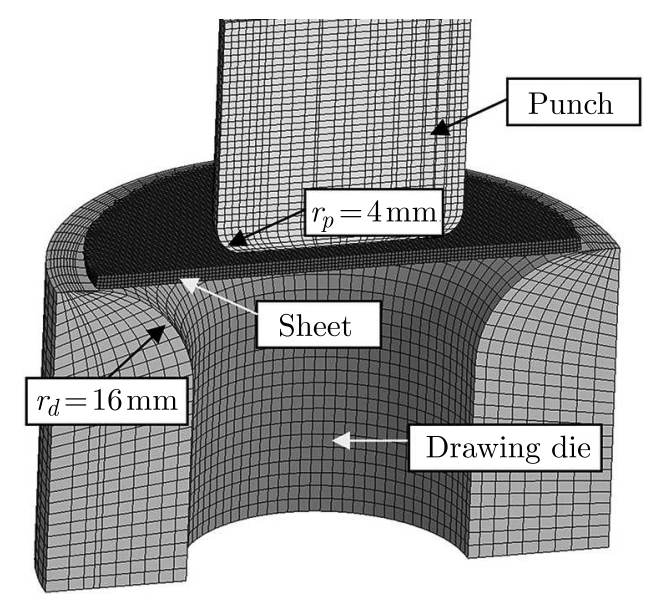

Fig. 5. A discrete model of the drawing process

Table 1. Acceptable reduction of the diameter in the drawing process for the ratio $D / g$ (Marciniak, 1998)

\begin{tabular}{|c|c|}
\hline$D / g$ & $m_{1}=d / D$ \\
\hline \hline 630 & 0.62 \\
\hline 400 & 0.60 \\
\hline 250 & 0.58 \\
\hline 160 & 0.56 \\
\hline 100 & 0.54 \\
\hline 63 & 0.52 \\
\hline 40 & 0.50 \\
\hline 25 & 0.48 \\
\hline
\end{tabular}


At first, analysis of the numerical drawing process without taking into account the history of stress and strain from the blanking has been performed. Thus, the initial values of stress and strain in all elements and nodes were 0 . What was left was only the mesh deformation of the disc as a result of blanking. Figure 6a shows a map of the equivalent stresses in the finished drawpiece. It can be observed that the values are rising from the bottom transition area through the side wall to the periphery. The maximum values of stresses during the drawing process occur at the periphery as a result of circumferential compressive stresses. Unevenness on the edge results from sheet discretization.

After the drawpiece forming simulation, a springback type analysis has been performed. It is analysis performed with the implicit integration method. It enables removal of all the nodes and elements of elastic strains, and it simulates the release of the product from the die. As a result of the removal of elastic strains, the product undergoes expansion, and its external diameter increases from $40 \mathrm{~mm}$ (Fig. 6a) to $40.13 \mathrm{~mm}$ (Fig. 6b). Also, the stresses occurring at the periphery of the drawpiece are reduced by approximately $25 \%$. Moreover, their location change. They occur in around half of the product height.
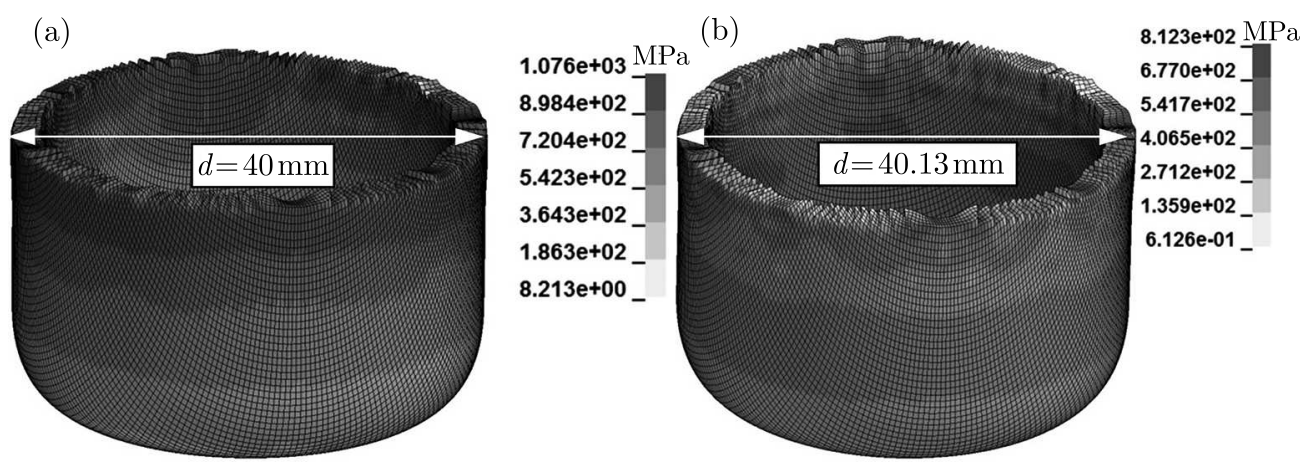

Fig. 6. Maps of the equivalent stress in the drawpiece without history: (a) before springback,

(b) after springback

\subsection{Deep drawing with the history of stress and strain}

To simulate the drawing process including the history of stress and strain, the disc after blanking and after springback analysis has been imported.To simulate the drawing process including the history of stress and strain, in the disc after blanking and after springback, analysis was performed taking into account the stress and strain values. All the conditions for the process, that is: diameters and radii remained unchanged. Figure 7a shows maps of the equivalent stress in the finished product. It can be seen that also in this case, the maximum stresses are located on the periphery of the product. However, the values are about $66 \%$ higher than in the case without including the history of stress and strain. In addition, the process of shaping the product proceeded in the same manner as in the previous case. The obtained diameter of the drawpiece corresponds to the internal die diameter, i.e. $40 \mathrm{~mm}$.

At the next stage, the springback analysis has been performed (Fig. 7b). The product behaved in a similar manner as in the case without including the history. The maximum stress value was migrated from the periphery to about half of the drawpiece height and decreased by $45 \%$. The diameter of the product was increased to $40.11 \mathrm{~mm}$, so that was almost the same as without including the stress and strain history.

\subsection{Comparision of two examples}

The next step is a detailed comparison of the drawpiece obtained without including the history of stress and strain with the drawpiece in which that history is included. Figure 8 shows 


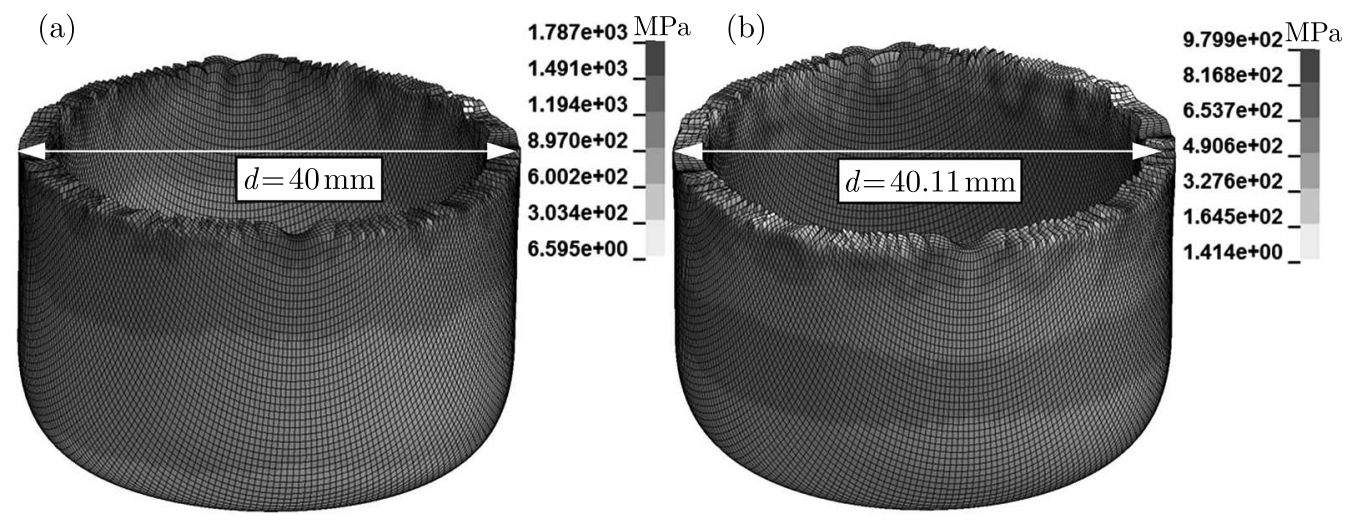

Fig. 7. Maps of the equivalent stress in the drawpiece with history: (a) before springback,

(b) after springback

the graph of the drawing force as a function of punch displacement for these two cases. The solid line represents the dependence with including the history. The dotted line represents the dependence without including the history. It can be observed that the two graphs are similar in characteristics. The differences are only in terms of the value, and there is a slight shifting. The fluctuations at the first stage of the graph are probably a result of discontinuous contact at the beginning of the drawing process. The maximum drawing force in the case with the history included is equal to $65 \mathrm{kN}$, without including the history it is less by about $7 \mathrm{kN}$. The difference in the value due to the fact that the disc has hardening at the periphery after blanking. This requires a greater force to deform it further. In both cases, the achievement of the maximum drawing force occurred at the punch displacement being equal to $25 \mathrm{~mm}$. This is the moment where there is the greatest peripheral drawpiece flange compression.

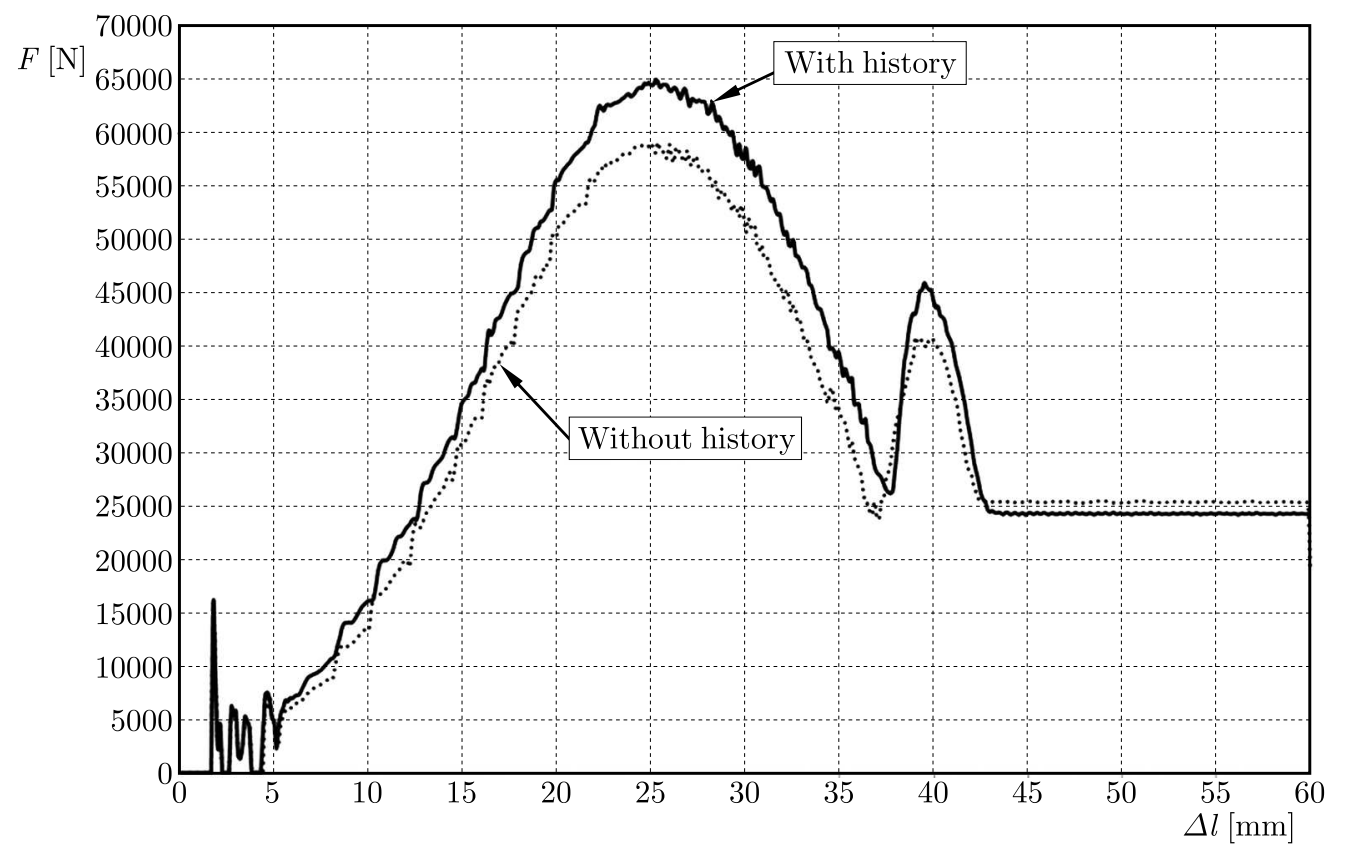

Fig. 8. Dependence of the drawing force from punch displacement

Figure 9 shows the deformation of the product for the maximum drawing force without including the history of stress and strain. After passing the maximum value of the drawing force, its value decreases until the periphery of the drawpiece is located directly between the die and the punch. At this point, the drawing force increases again, in the case with including 
the history up to $46 \mathrm{kN}$, and for the case without the history up to $41 \mathrm{kN}$. This increase results directly from the clearance between the die and the punch. In this case, this is adopted less intentionally to observe the effect it would have on the formation of the product. This moment is illustrated in Fig. 10. The final step of drawpiece forming can be observed, which consists in compression of the drawpiece peripheral to the size of the clearance equal to $2.5 \mathrm{~mm}$. An improper selection of the clearance may cause at this point, during the experiment on the hydraulic press, jamming and damage of the tools.

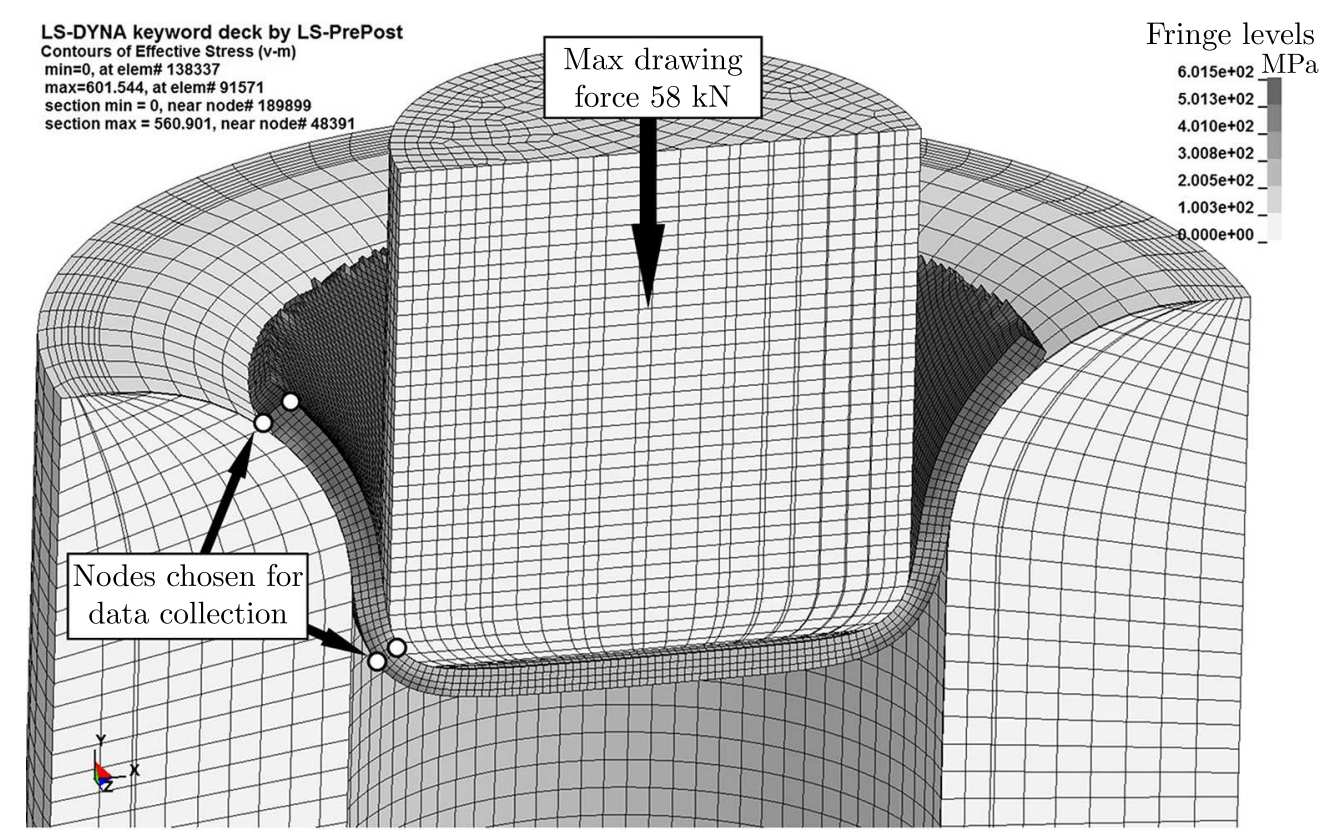

Fig. 9. Deformation of the product for the maximum drawing force without the history of stress and strain

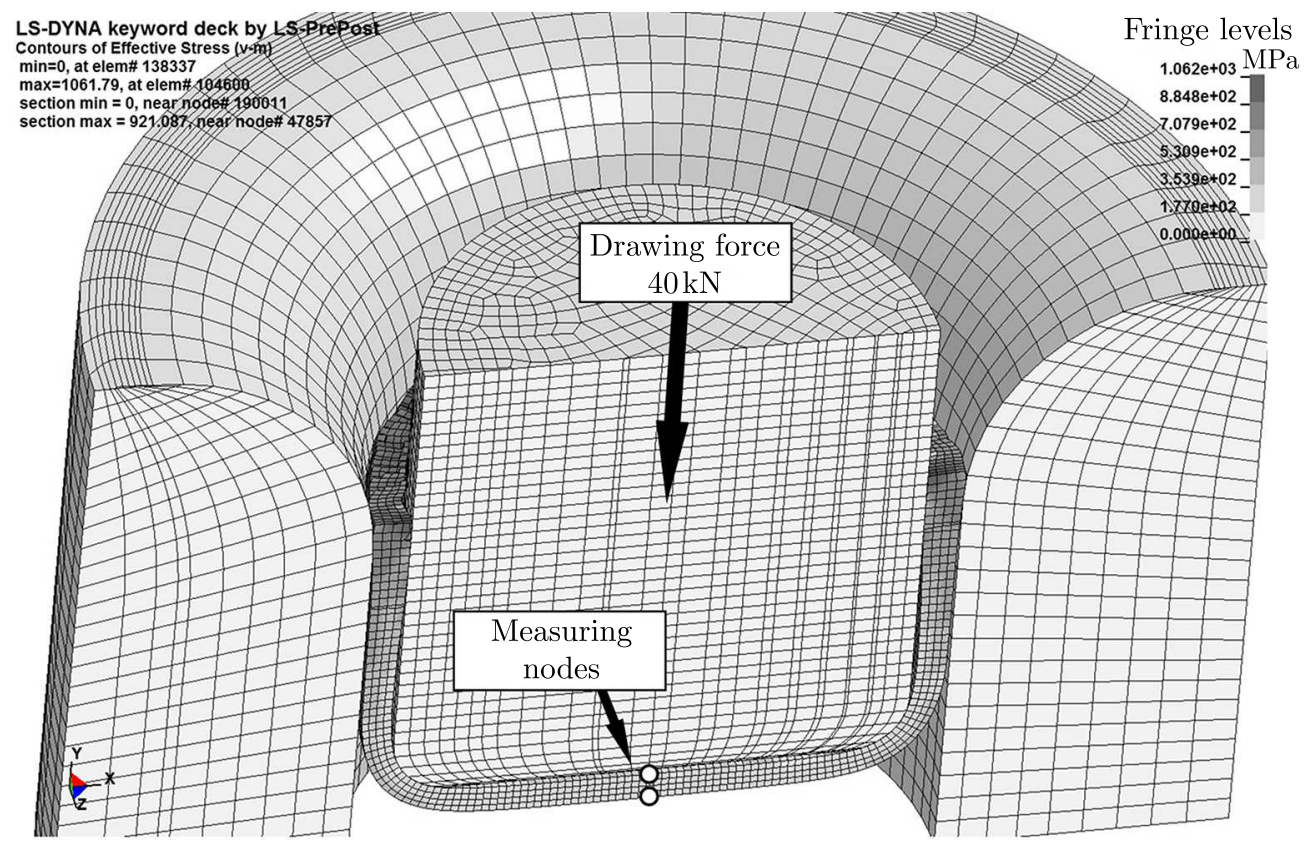

Fig. 10. Final drawpiece formation without the history of stress and strain 
Apart from the drawing force, it is also possible to compare products in terms of their geometry and shape. Namely, the two drawpieces sheet thicknesses in characteristic locations and their total heights are compared. The reading is performed between the same nodes in each case. This proceeding allows us to eliminate errors of the mesh deformation, or other reading inaccuracies. Figure 11a shows a graph of the sheet thickness changes at the drawpiece periphery as a function of the punch displacement. It can be observed that the drawpiece, almost from the beginning of the drawing process with the history included, has a greater thickness at its periphery. This is due to the fact that the compressive circumferential stress during the process is cumulative with the stress after blanking. The compression of such a tensioned material is difficult, therefore, some of the material moves inwards or outwards and causes enlargement of the sheet thickness. The maximum difference in the thickness of the sheet metal at the periphery occurs before the punch and reaches a $40 \mathrm{~mm}$ displacement. For the drawpiece with the history included, the thickness is equal to $2.77 \mathrm{~mm}$, whereas without the history is equal to $2.68 \mathrm{~mm}$. This situation occurs immediately before the moment the periphery is compressed between the punch and the die, as shown in Fig. 10. Then, after forming the drawpiece walls to the cylindrical form, the edge thickness is nearly identical for the two cases and it is $2.54 \mathrm{~mm}$ for the case with and $2.53 \mathrm{~mm}$ without the history. The value at end in both cases that is larger than the clearance value equal to $2.5 \mathrm{~mm}$ is due to friction. The nodes between which the reading is made are not in a perfectly horizontal position. As a result of sliding friction on the die and static on the punch, its minor displacement occurs.

Another dimension that is compared in both cases is the sheet thickness measured at the center of the drawpiece bottom. The reading has been conducted in the same manner as before. What is measured during the whole process is the distance between nodes, at the outer and inner surface, in the middle of the disc. The reading results are presented in the graph in Fig. 11b. The reading starts from the initial value of the sheet thickness of $2.01 \mathrm{~mm}$. Then, during the process, small thinning follows in the bottom sheet. In the case where the history of stress and strain is included, the thickness in the final product is $1.96 \mathrm{~mm}$, whereas without history it is $1.97 \mathrm{~mm}$. A reduction in the sheet thickness in this area is due to radial extention of the bottom during the drawing process. This is often so slight thinning that besides the reading of the simulation result it is very difficult to observe. Also in this case, the difference on the level of $0.01 \mathrm{~mm}$ is only a general value, that the history of stress and strain causes a marginal effect on the change in the thickness of the bottom drawpiece.

(a)

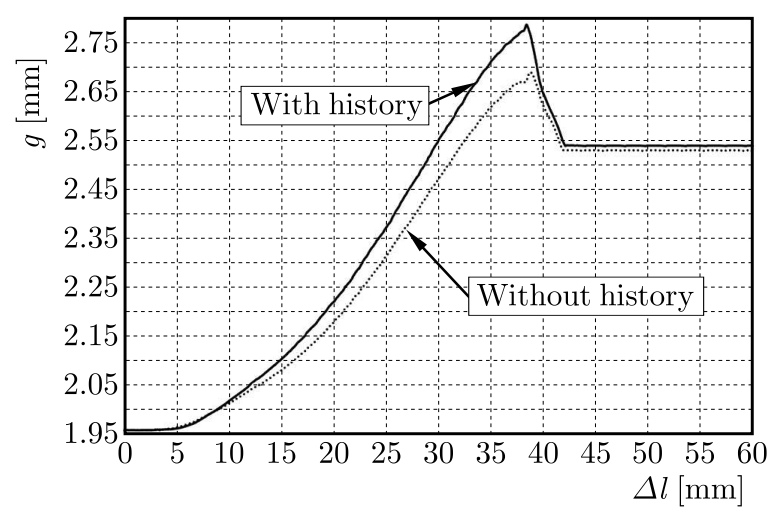

(b)

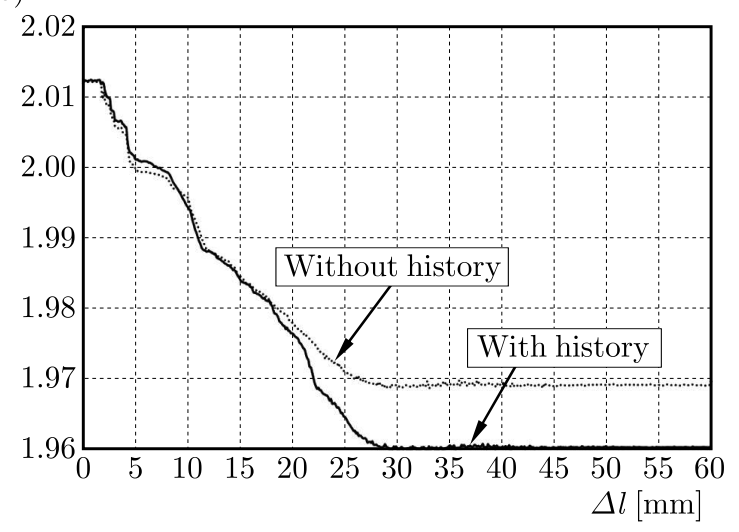

Fig. 11. Dependence of: (a) edge thickness from punch displacement, (b) bottom thickness from punch displacement

Much more important is the reading of the sheet metal thickness on the curved edge of the drawpiece, where the bottom passes into the cylindrical wall. An improper selection of the clearance or a too large diameter of the output disc in relation to the internal die diameter may 
cause bottom break off during the drawing process. The bottom break off occurs most frequently in the area where the bottom passes into the cylindrical wall, because there occurs the greatest thickness reduction of the sheet. The dependence of the sheet thickness changes as a function of the punch displacement is plotted in Fig. 12a. The reading starts from the initial value of the sheet thickness of $2.01 \mathrm{~mm}$. Larger sheet thinning occurred when including the history of stress and strain. The sheet thickness decreases to $1.74 \mathrm{~mm}$. This is due to significant hardening of the drawpiece periphery, which makes entry into the die difficult, and this, in turn, promotes thinning of the sheet metal at its rounding. In the case whithout the history included, the sheet thickness in this area is $1.76 \mathrm{~mm}$. Similarly, as for measuring of the bottom thickness, these differences are difficult to observe and are measured in a traditional experiment.

The last element which is compared is the drawpiece height. The dependence, as a function of the drawpiece height from the punch displacement, is shown in the graph in Fig. 12b. Both characteristics are almost identical to the punch displacement of $40 \mathrm{~mm}$. The last stage of the drawing process is from the displacement $40 \mathrm{~mm}$ where a noticable difference between the heights are plotted in Fig. 12c. The drawpiece height shaped with the history included is equal to $26.21 \mathrm{~mm}$, whereas without the history it is $25.96 \mathrm{~mm}$. Marginally larger height of the product with the history included may result directly from the hardening of the drawpiece periphery. This hardening, in turn, influences substantial thickening of the periphery. This, in turn, at the final stage makes the transition difficult between the die and the punch. This affects the greater flow on the sidewall, bottom and the area where the bottom passes into the cylindrical wall. Similarly as in the reading of the sheet thickness at the periphery of drawpiece, in the bottom and rounding, the differences are minor and difficult to measure in a traditional experiment. Numerical analysis allows one to detect these slight size differences.

(a)

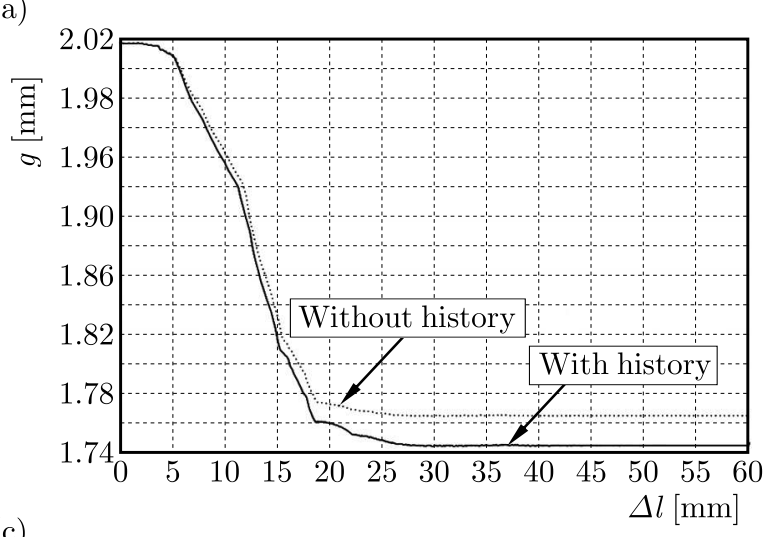

(c)

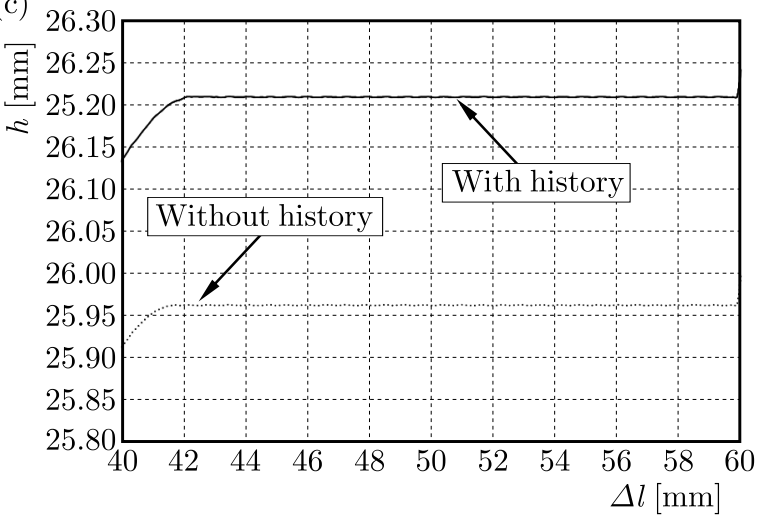

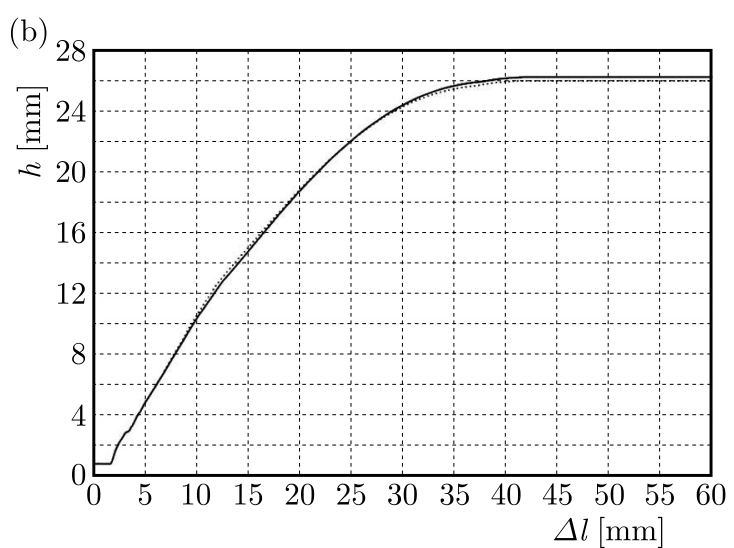

Fig. 12. Dependence of: (a) rounding thickness from punch displacement, (b) drawpiece height from punch displacement, (c) drawpiece height from punch displacement (zoom) 


\section{Conclusions}

In this paper, the results of numerical complex modelling of the blanking and drawing processes have been compared. After blanking simulation, a springback analysis has been conducted. Then, two analyses of drawing were carried out and the results were compared. One with the history included of stress and strain after the blanking process, and the other one without that history. The comparative elements between the analyses were: the drawing force as a function of punch displacement, changing of the sheet thickness at the bottom, at the periphery and the rounding, and also the final drawpiece height. In addition, the equivalent stress distributions in the drawpieces were compared.

It was observed that including the history in the drawing process caused an increase of the maximum drawing force by $12 \%$. There were also marginal differences in the drawpiece dimensions, which were impossible to measure with traditional methods during the experiment. The sheet thickness on the periphery, until the whole drawpiece had been formed, was higher by $3.4 \%$. The drawpiece bottom thickness decreased by $0.5 \%$. Also, the rounding thickness decreased by $1.1 \%$. The product with the history included was $1 \%$ higher than without the history. The most significant difference was noted in the stress values. The drawpiece with the history included had a $66 \%$ higher stress values in the last step of the analysis. After the springback analysis, the stress values was higher by $20.7 \%$ than in the analysis without the history.

\section{References}

1. Bohdal L., 2015, Application of FEM and vision-based methods to analysis of shearing processes in the aspect of scrap reduction, Annual Set the Environment Protection, 17, 90-103

2. Bohdal L., Kukielka L., 2014, Application of variational and FEM methods to the modelling and numerical analysis of guillotining process for geometrical and physical nonlinearity, Mechanics, 20, 2, 197-204

3. Bohdal L., Kukielka L., Kukielka K., Kulakowska A., Malag L., Patyk R., 2014, Three dimensional finite element simulation of sheet metal blanking process, Applied Mechanics and Materials, 474, 430-435

4. Gambin W., KowalczyK K., 2003, Plasticity of Metals (in Polish), Warsaw University of Technology Publishing House

5. Jemiolo S., Gajewski M., 2014, Hyperelasto-Plasticity (in Polish), Warsaw University of Technology Publishing House

6. Kaldunski P., 2009, Modelling and simulation forming of circularly-symmetrical drawpieces without flange with the regard for the geometrical and physical nonlinearity (in Polish), PhD Thesis, Koszalin

7. Kaldunski P., Kukielka L., 2014, Numerical analysis and simulation of drawpiece forming process by Finite Element Method, Applied Mechanics and Materials, 474, 153-158

8. Kim T.H., Olver A.V., 1998, Stress history in rolling-sliding contact of rough surfaces, Tribology International, 31, 12, 727-736

9. Marciniak Z., 1998, Technology of the Drawpieces and die Design. Notebook Problems (in Polish), Technical Center A. Marciniak, Warszawa

10. Nagasekhar A.V., Tick-Hon Yip, Li S., Seow H.P., 2006, Stress and strain histories in equal channel angular extrusion/pressing, Materials Science and Engineering: A, 423, 143-147

11. Simo J.C., Hughes T.J.R., 1998, Computational Inelasticity, Springer-Verlag, New York 
12. Trzepiecinski T., Gelgele H.L., 2011, Investigation of anisotropy problems in sheet metal forming using finite element method, International Journal of Material Forming, 4, 357-369

13. Urriolagoitia-Sosa G., Romero-Angeles B., Hernandez-Gomez L.H., Torres-Torres C., Urriolagoitia-Calderon G., 2011, Crack-compliance method for assessing residual stress due to loading/unloading history: Numerical and experimental analysis, Theoretical and Applied Fracture Mechanics, 56, 188-199

14. YAO H., CAO J., 2001, Assessment of corner failure depths in the deep drawing of 3D panels using simplified 2D numerical and analytical models, Journal of Manufacturing Science and Engineering, 123, 248-257

15. Zienkiewicz O.C., TAYlor R.L., 2006, The Finite Element Method for Solid and Structural Mechanics, Elsevier, 6th edition

Manuscript received Septemer 30, 2015; accepted for print December 21, 2017 